
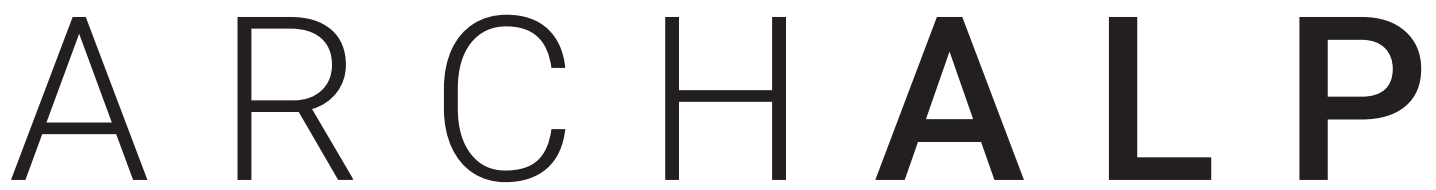

Rivista internazionale di architettura e paesaggio alpino / Revue internationale d'architecture et de paysage dans les Alpes / Internationale Zeitschrift für Alpine Architektur und Landschaft / Revija za alpsko arhitekturo in pokrajino / International journal of alpine architecture and landscape 


\title{
Legare, incorniciare, sottolineare, amplificare: due progetti per gli spazi pubblici di insediamenti storici trentini
}

Linking, framing, underlining, amplifying: two projects for the public spaces of historic Trentino settlements

\begin{abstract}
The small villages in rural areas of Trentino often share similar settlement principles (of Latin-Romansh origin) and destinies.

Their dense cores suffer from a phenomenon of consistent abandonment that causes the aweakening of the social structure and an increasing obsolescence of the buildings.

The two projects of Castelfondo (Municipality of Borgo d'Anaunia) and Bolciana (Municipality of Tre Ville) are to be considered in this general context, in two small villages that felt the need to regenerate the public space to provide new places for social gathering and new facilities to the community.

In Castelfondo, the abandonment of a building is the starting point of the project: instead of a decaying building, three small public spaces offer a new gathering place for the community.

In Bolciana, the need to have spaces that would improve the quality of the existing places led to a project made of small artifacts in four squares.

The two projects try to demonstrate that with a careful approach by the municipalities, the needs and problems of locations that are far from large tourist flows and main infrastructures can become fertile opportunities to reinvent spaces and meet emerging needs.
\end{abstract}

\section{Mirko Franzoso, Mauro Marinelli}

They founded franzosomarinelli, an architecture studio based in Cles (Trentino / Südtirol, Italy). The studio deals with architectural research and design at different scales, looking further into the issues related to contemporary Alpine architecture.
Keywords

Dolomites, villages, contemporary architecture, regeneration, public spaces.

Doi: 10.30682/aa2107p 
In apertura

Rigenerazione degli spazi pubblici di

Bolciana, Treville, Provincia di Trento, 2020 (foto Mariano

Dallago).

Figg. 1-2 Nuove piazze e spazi pubblici a Castelfondo, Borgo d'Anaunia, Provincia di Trento, 2020 (foto Mariano Dallago)
I piccoli insediamenti che punteggiano i territori rurali trentini spesso sono accomunati da principi insediativi simili (di matrice latino-romancia) e da destini condivisi.

I nuclei densi hanno fenomeni di abbandono sempre più consistenti con un conseguente impoverimento del tessuto sociale e una sempre più emergente obsolescenza dei manufatti.

In questo contesto generale si inseriscono i due progetti di Castelfondo (Comune di Borgo d'Anaunia) e di Bolciana (Comune di Tre Ville), due piccoli villaggi che hanno sentito la necessità di rigenerare lo spazio pubblico per dotare la comunità di nuovi luoghi di aggregazione e nuovi servizi.

A Castelfondo il tema dell'abbandono dei manufatti diventa l'occasione del progetto.
Il Comune si è trovato con la necessità di demolire un edificio fatiscente destinato presto a soccombere sotto i colpi della neve e del tempo. Questa urgenza non è stata subita in maniera passiva dalla comunità ma è stata interpretata come occasione fertile e imprevista per rigenerare il tessuto insediativo e dotare il paese di nuovi spazi dove ritrovarsi e riconoscersi.

Il progetto, che in qualche modo vuole risarcire l'assenza dell'edificio, si articola attorno a una serie di muri che, con la loro massività, dialogano con gli edifici circostanti e ricordano l'edificio preesistente, definendo tre piazze che si accostano ai livelli del suolo esistente.

In questo modo tre spazi pubblici diversi definiscono un sistema fluido di stanze che si affacciano l'u-

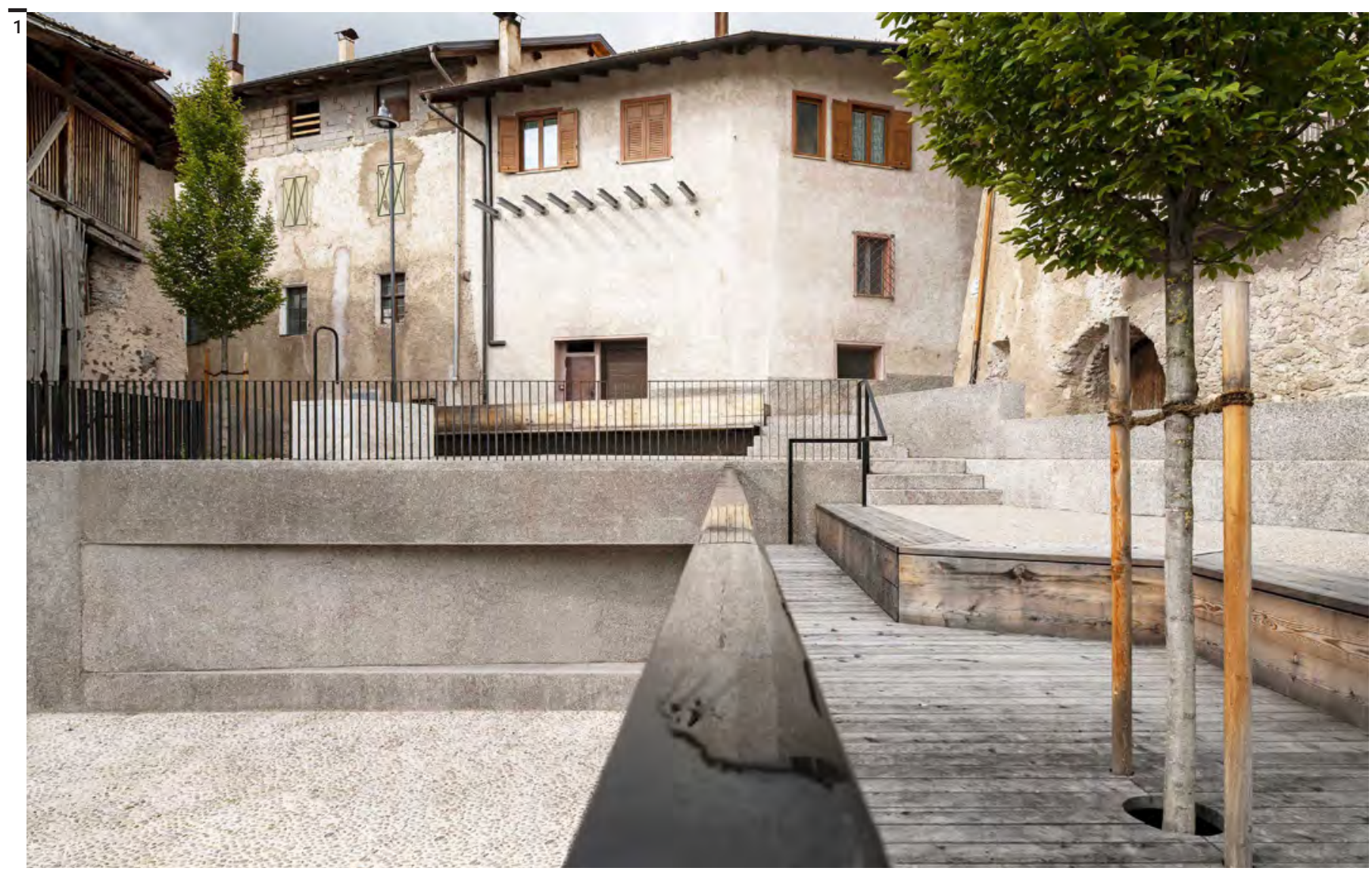




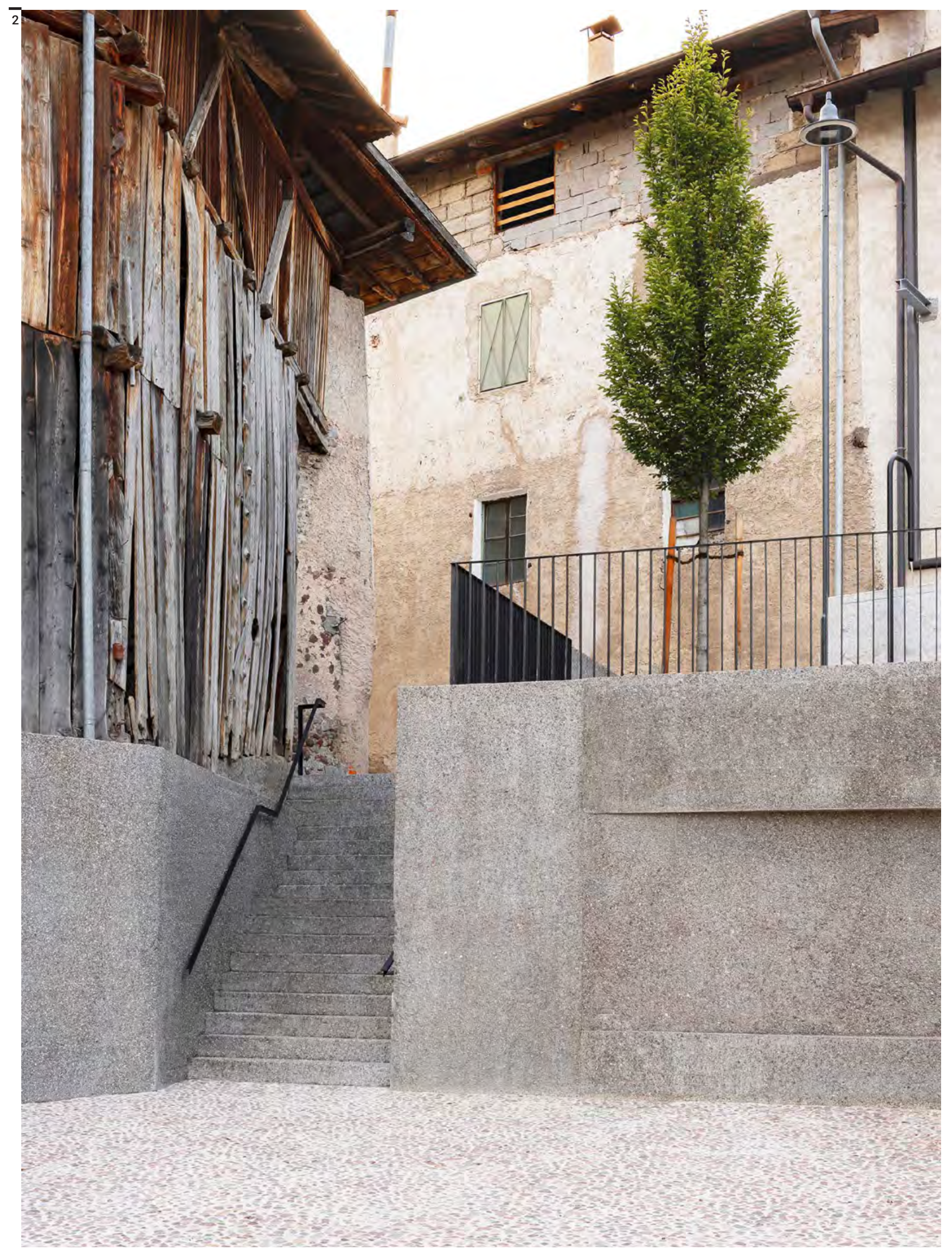




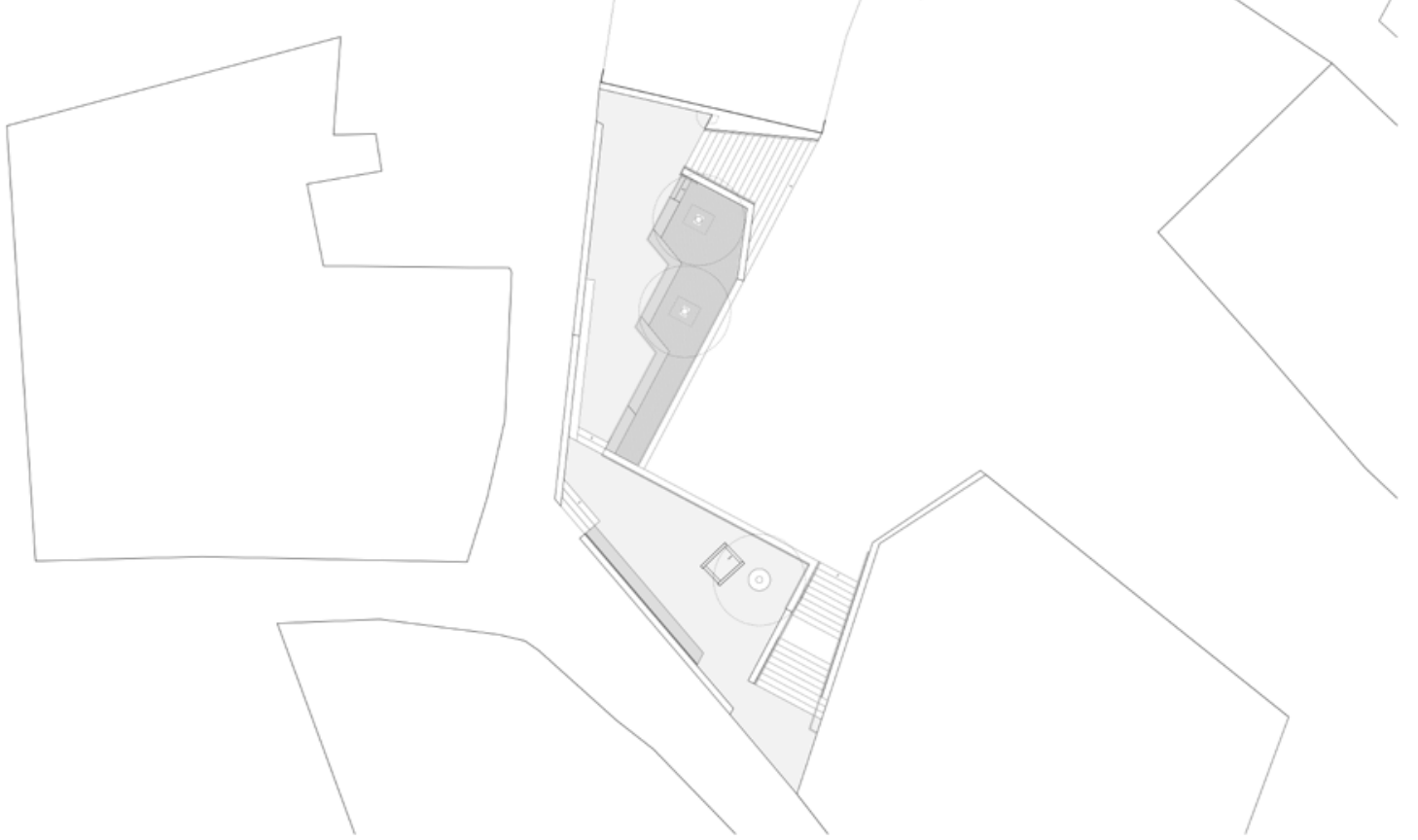

na sull'altra coinvolgendo gli edifici circostanti con le loro tessiture e materiali.

Le scale, collocate ai bordi degli spazi, permettono il passaggio tra le diverse quote ma soprattutto si configurano come dispositivi percettivi del luogo, inquadrando viste e generando compressioni e dilatazioni dello spazio.

Le singole piazze hanno così caratteri differenti: quella inferiore è una sorta di palcoscenico dove la comunità può ritrovarsi per le festività e le rappresentazioni teatrali; la piazza intermedia si affaccia sulla prima ed è modellata come una grande seduta realizzata con il morbido legno di larice locale dove poter sostare anche in piccoli gruppi di persone; la terza ospita una seduta dove poter godere, in un'atmosfera intima e famigliare, della piccola fontana costruita riciclando vecchie lastre abbandonate di una precedente fontana che giacevano a terra dimenticate in una delle proprietà comunali.

Il materiale scelto è di particolare importanza perché cerca un legame con il luogo a diverse scale: è un calcestruzzo con sabbia e inerti di porfido sbrecciati che, lavato subito dopo il getto, rivela le pietre in superficie. La superficie ruvida e vibrante si accorda con le

Fig. 3

Nuove piazze e spazi pubblici a

Castelfondo, Borgo

d'Anaunia, Provincia

di Trento, 2020,

planimetria. degli edifici circostanti grazie ai quali genera un'atmosfera semplice, calda e sospesa nel tempo.

L'architettura tenta quindi di regalare spazi che una comunità possa sentire propri e che abbiano un carattere famigliare, intimo, accogliente.

A Bolciana invece la necessità era quella di avere spazi con differenti usi che fossero in grado di migliorare la qualità dell'abitare nella speranza di limitare lo spopolamento o attrarre nuovi abitanti.

La qualità dell'insediamento storico e le necessità di una sua rigenerazione hanno subito posto un'appassionante sfida progettuale, nella convinzione che il progetto dovesse porsi in continuità con l'esistente, dichiarando la contemporaneità dell'intervento e rielaborando l'identità specifica e preziosa dei luoghi.

La strategia progettuale nasce dalla lettura dell'abitato storico che, nel suo carattere denso e compatto, appare come una sequenza di "stanze all'aperto" che gravitano lungo l'asse della via principale.

La matrice latino-romancia dell'insediamento è forte e configura un nucleo particolarmente denso di manufatti che si organizzano sul ripido suolo definendo spazi aperti sorprendenti, talvolta racchiusi ed intimi, talvolta aperti e protesi sul paesaggio.

Partendo da questa condizione identitaria contestuale il progetto tenta di esaltare la natura degli spazi dell'insediamento articolando il rapporto tra la via e le singole "stanze all'aperto" attraverso la ri- 
$\overline{4}$

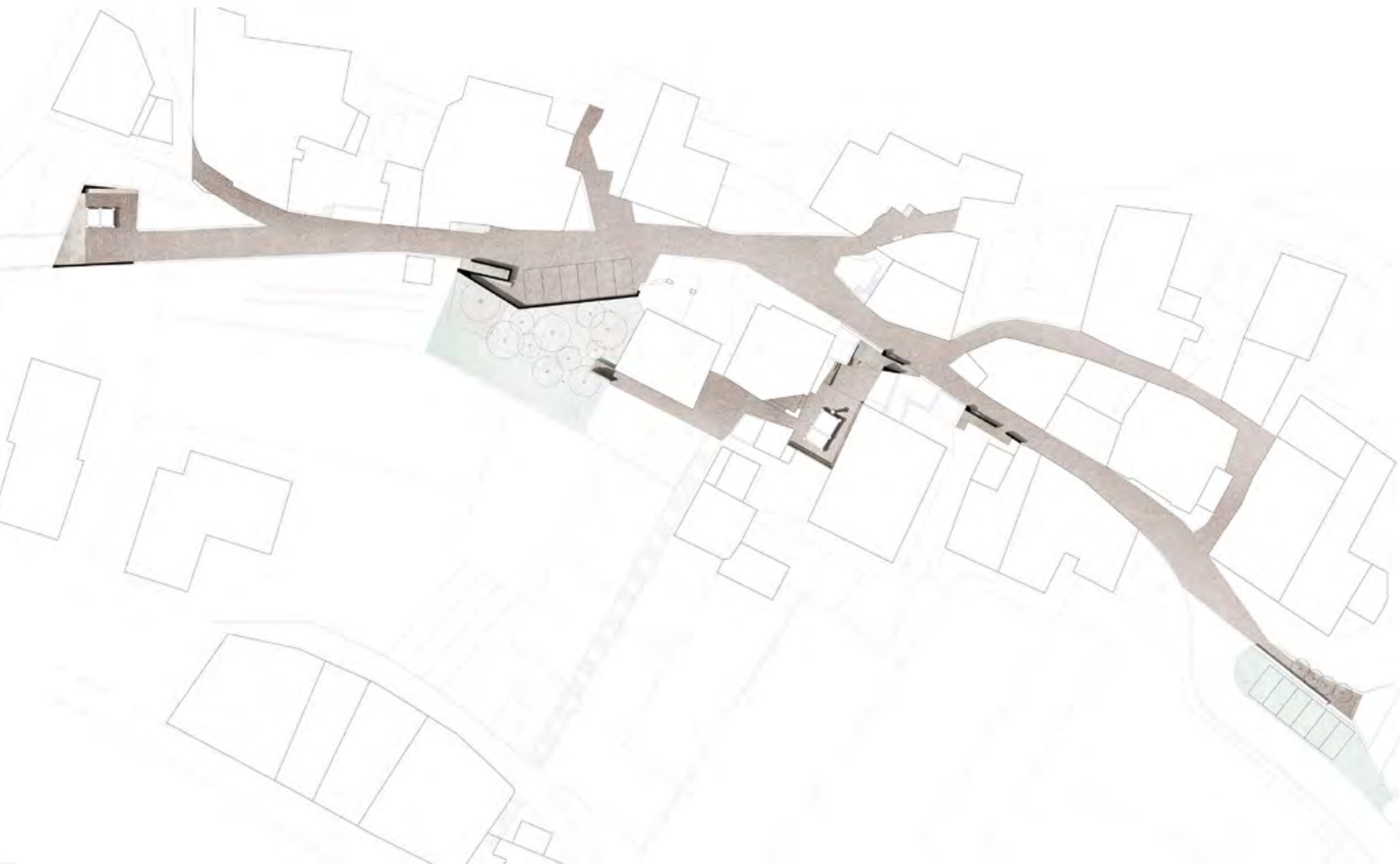

definizione dei bordi dei singoli spazi e l'articolazione delle soglie che ne definiscono gli accessi.

Lavorando in quattro principali punti, come una sorta di agopuntura, piccoli manufatti in ruvido calcestruzzo bianco bocciardato dialogano con le massicce pietre dei muri preesistenti e con gli intonaci ruvidi degli edifici circostanti rapportandosi con il ripido suolo in maniera sempre differente e pro- muovendo ogni volta usi diversi dello spazio (con una fontana, una seduta, una scala, un portico coperto, un parcheggio).

Le loro geometrie sono dettate dal contesto in un rapporto di dialogo fertile che pone il progetto in una condizione di rispettosa continuità.

Le singole stanze acquistano identità autonome partecipando però alla definizione di un sistema unita-

Fig. 4

Rigenerazione degli spazi pubblici di

Bolciana, Treville, Provincia di Trento, 2020, planimetria.

Fig. 5

Rigenerazione degli spazi pubblici di Bolciana, Treville, Provincia di Trento, 2020 (foto Mariano Dallago)

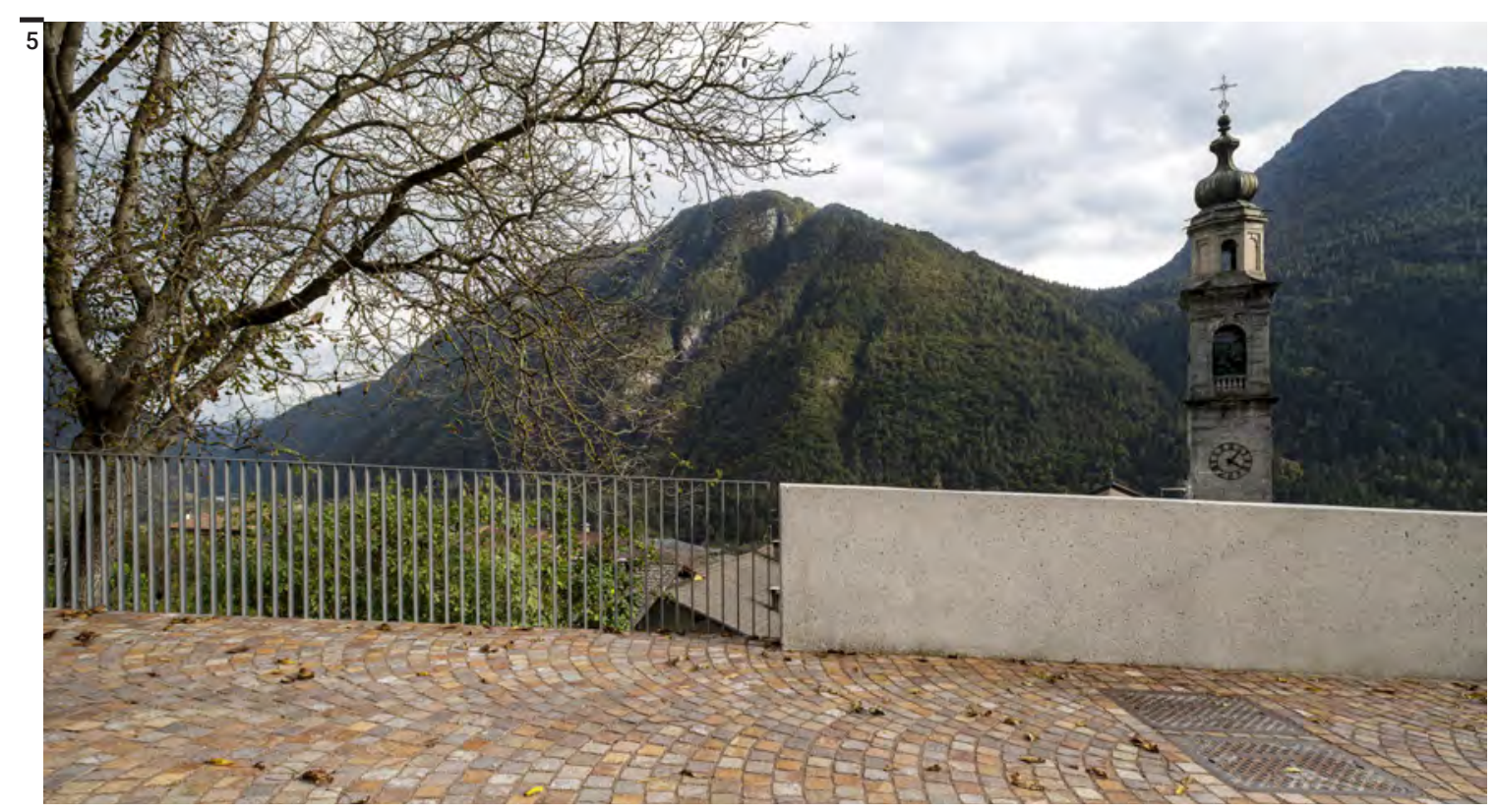


rio alla scala del villaggio ripercorrendo in qualche modo l'identità dell'insediamento stesso che, seppur composto di singoli episodi architettonici, compone un grande manufatto collettivo coerente e unitario. I piccoli interventi, nelle loro geometrie complesse, vengono disegnati da ciò che li circonda seguendo allineamenti e geometrie preesistenti tentando di interpretare ogni accidente locale quale occasione progettuale. Ospitando funzioni diverse, definendo spazi con caratteri specifici, modellando la luce in maniera inattesa, essi generano rapporti sorprendenti con il paesaggio che, tra gli edifici, appare con l'affascinante forza dei boschi e delle vette. Gli spazi che stanno fra gli edifici ed i piccoli manufatti in cemento diventano pause di un'unica composizione: parapetti leggeri in metallo si contrappongono alla massività del calcestruzzo e della pietra richiamando il dualismo tra elementi massivi e leggeri esistente negli edifici locali.

Figg. 6-8 Una pavimentazione di porfido si configura come Rigenerazione degli spazi pubblici di Bolciana, Treville, Provincia di Trento, 2020 (foto Mariano Dallago). un piano su cui i singoli elementi dialogano e interpretano i differenti ruoli che il contesto richiede, tra linguaggi contemporanei e caratteri tradizionali.

Nei mesi seguenti alla realizzazione la popolazione si è appropriata degli spazi che oggi appaiono attivi e riconquistati, tra anziani che usano nuovamente le fontane per il lavaggio dei panni, e bambini che reinventano, attraverso il gioco, luoghi e manufatti. Inoltre alcuni abitanti hanno in qualche modo "imitato" le tecniche costruttive proposte dal progetto per alcuni piccoli interventi privati: un piccolo segno, ma molto significativo, di come gli elementi introdotti con il progetto siano stati accolti e siano ora parte dell'identità della comunità che abita il nucleo di Bolciana.

I due progetti stanno dunque dimostrando quanto le necessità e i problemi che talvolta possono affliggere luoghi lontani dai grandi flussi turistici e da grandi infrastrutture di attraversamento, grazie ad un approccio attento da parte delle amministrazioni possono diventare fertili opportunità di reinvenzione degli spazi e di risposta a nuove esigenze. Possono dunque offrire occasioni inattese per rigenerare luoghi il cui potenziale è in attesa.

La speranza è che l'architettura e i progetti siano stati in grado di incaricarsi di queste nuove necessità dando corpo a luoghi che possono ospitare le comunità e i loro destini costellati di occasioni da cogliere e rinnovate speranze.

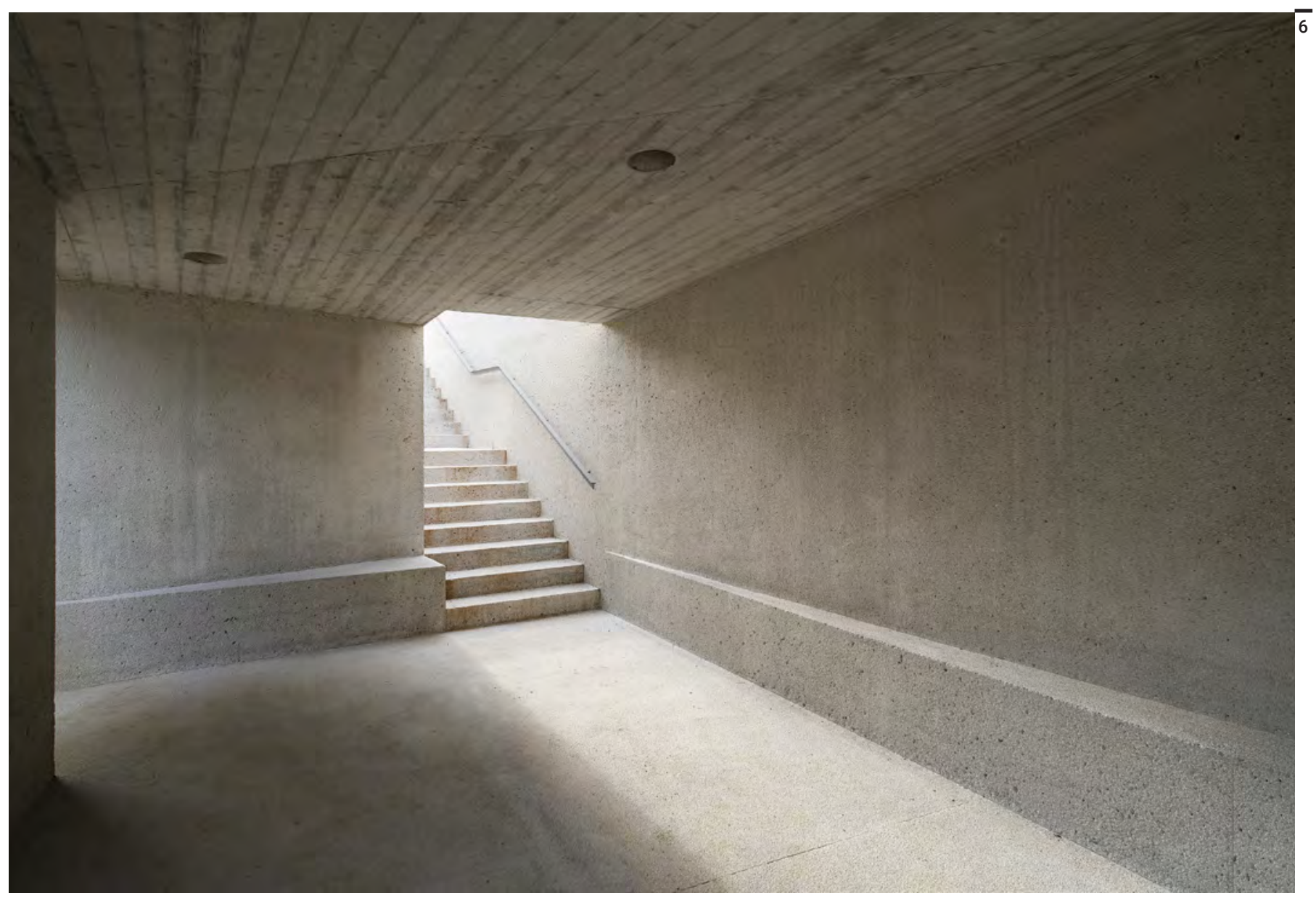



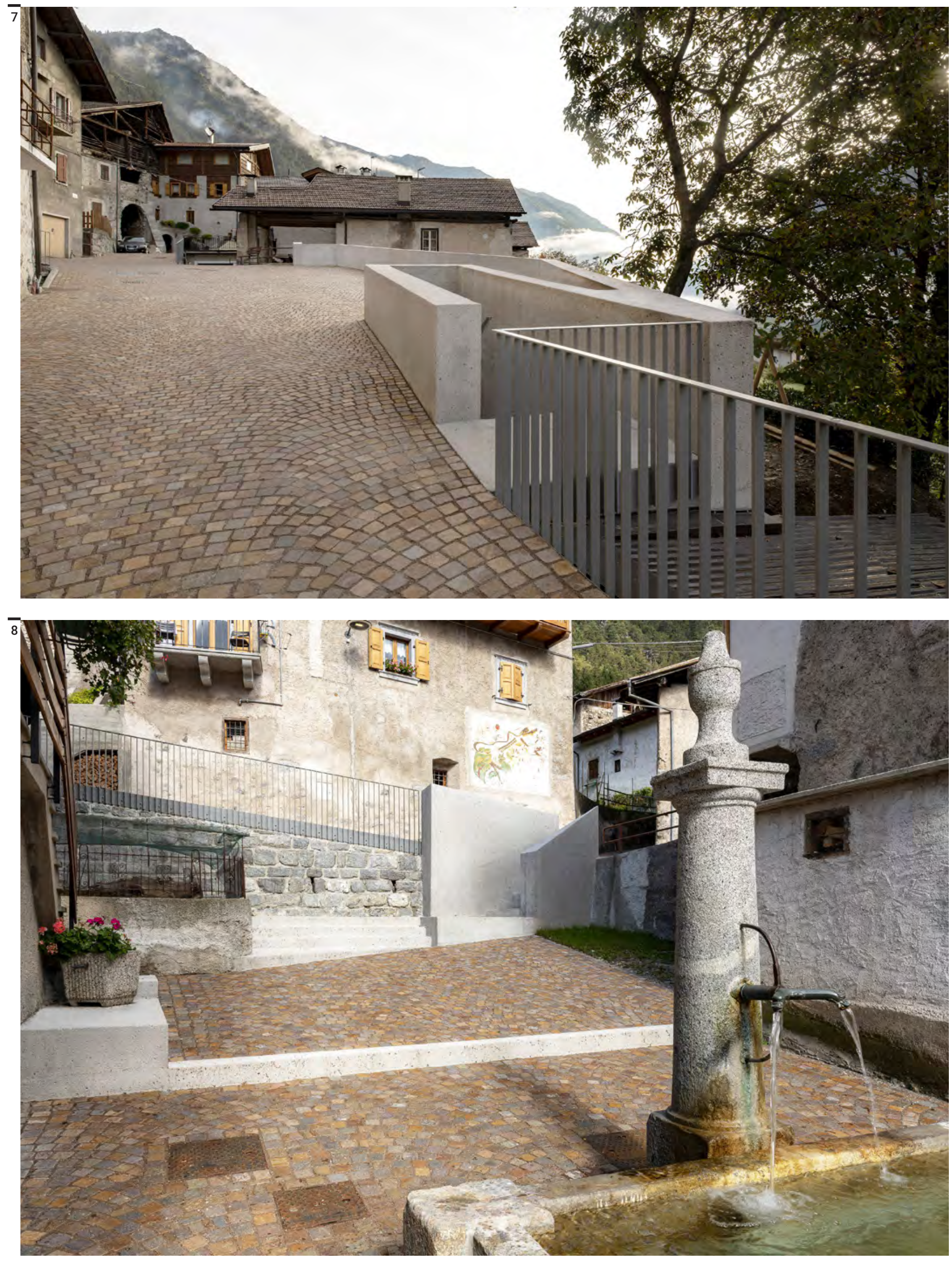


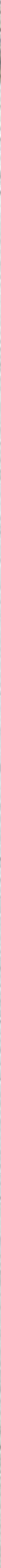

